\title{
Uniportal video-assisted thoracoscopic segmentectomy
}

\author{
Luis Angel Hernandez-Arenas ${ }^{1}$, Rushmi D. Purmessur ${ }^{1}$, Diego Gonzalez-Rivas ${ }^{2}$ \\ ${ }^{1}$ Department of Thoracic Surgery, Birmingham Heartlands Hospital, NHS Trust Foundation, Birmingham, UK; ${ }^{2}$ Department of Thoracic Surgery, \\ Minimally Invasive Thoracic Surgery Unit (UCTMI), Coruna Hospital, Coruna, Spain \\ Correspondence to: Diego Gonzalez-Rivas. Department of Thoracic Surgery, Minimally Invasive Thoracic Surgery Unit (UCTMI), Coruna Hospital, \\ Coruna, Spain. Email: diego.gonzalez.rivas@sergas.es.
}

\begin{abstract}
Uniportal video-assisted thoracoscopic surgery (VATS) segmentectomy is a technically more complex procedure than uniportal VATS lobectomy, since a detailed comprehension of the segmental anatomy is required. Anatomic sublobar resection can achieve outcomes equivalent to lobectomy in selected patients with stage IA non-small cell lung carcinoma (NSCLC). In this paper we describe our clinical experiences and introduce the technical details of uniportal VATS segmentectomy, including expertise advice ("tips and tricks") in patient selection, positioning and incisions and technical highlights of the most common types of segmentectomies. Uniportal VATS segmentectomy is a demanding technique, safe and feasible in selected patients with good results in the literature that seems to be an acceptable alternative to conventional VATS or open thoracotomy when the surgeon completed the training period and learning curve.
\end{abstract}

Keywords: Video-assisted thoracoscopic surgery (VATS); segmentectomy; uniportal; hook wire

Submitted Feb 06, 2018. Accepted for publication Feb 09, 2018.

doi: $10.21037 /$ jtd.2018.02.47

View this article at: http://dx.doi.org/10.21037/jtd.2018.02.47

\section{Introduction}

Uniportal video-assisted thoracoscopic surgery (VATS) is a novel approach that is currently becoming increasingly popular worldwide (1-3). It has been proven to be a feasible and safe technique in expert hands with similar outcomes as compared to the other approaches, with the advantage of having only one incision (4-6).

With recent advances in computed tomography (CT) imaging technology, the diagnoses of small lung nodules, ground glass lesions and non-solid lung cancers has been increasing (7-9). When such lesions are located deep within the lung parenchyma and R0 complete resection is required, segmentectomy is an alternative.

Segmentectomy is a technically more complex procedure than lobectomy, since a detailed comprehension of the segmental and subsegmental anatomy is required. Recent trials have shown that anatomic sublobar resection can achieve outcomes equivalent to lobectomy in selected patients with stage IA non-small cell lung carcinoma (NSCLC) (10-12). To be able to execute a uniportal VATS segmentectomy, we propose that the surgeon should have already passed the uniportal VATS learning curve and visited a high volume training centre where they perform segmental anatomic resections to become familiarised with the anatomy, different approaches and various tips and tricks. We recommend starting with easy selected cases (e.g., trisegmentectomy, lingulectomy or dorsal segment), including patients with clear fissures.

Segmentectomy by uniportal VATS approach requires expert knowledge of the anatomic structures and the correct and thorough interpretation of the images from the CT scan. At the moment of performing the segmental anatomical resection, we highly recommend a high resolution CT scan with $3 \mathrm{D}$ reconstruction (Figure 1) where possible to assess the lateral, the axial and the sagittal views in order to firstly recognize in which segment the lesion that needs to be dissected is located, and secondly recognise the segmental anatomical structures (including veins, arteries and bronchus) and identify the anatomic variations, for better surgical planning and to avoid technical faux pas and complications in order of planning the surgery better.

The segmental resection should always include the arterial, venous and bronchial stumps. In this section, we 

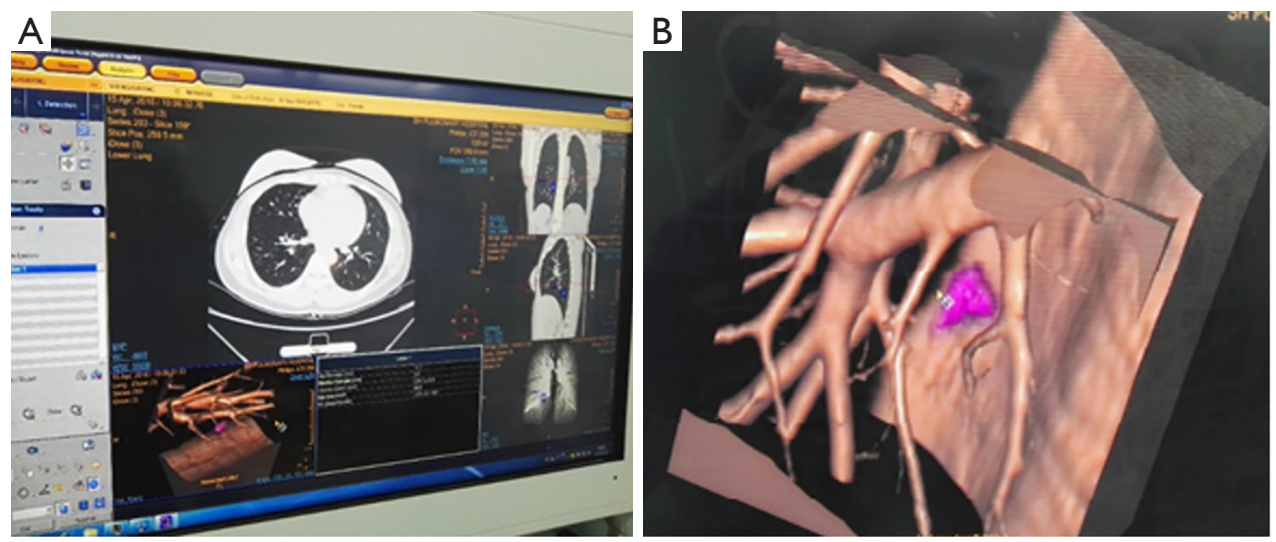

Figure 1 Image shows the CT-scan 3D reconstruction available inside the operating room. CT, computed tomography.

Table 1 Impart knowledge and expertise for uniportal VATS segmentectomy

Uniportal VATS learning curve passed

High resolution $\mathrm{CT}$ scan with $3 \mathrm{D}$ reconstruction with access in the operating room

Expert knowledge of the segmental and subsegmental anatomy and the anatomic variations

Use of double lumen tube with patient in the lateral decubitus position (using always the same positioning method)

VATS instruments set

Dissecting using bimanual dissection

Always include the arterial, venous and bronchial stumps

Use inflation/deflation method before dividing bronchus and intersegmental fissure

Use of CT-guided hook wires to locate the deep lesions

VATS, video-assisted thoracoscopic surgery; CT, computed tomography.

describe our clinical experiences and introduce the technical details of uniportal VATS segmentectomy, including expertise advice ("tips and tricks") (Table 1).

\section{Surgical technique}

\section{Approach}

\section{Patient selection}

Our indications for segmental lung resection include: tumours less than $2 \mathrm{~cm}$ in diameter without $\mathrm{N} 1$ or $\mathrm{N} 2$ disease when the tumour is completely contained with the anatomic boundaries of the segment, small ground glass opacities $<2 \mathrm{~cm}$, or a benign lung disease resectable through segmentectomy, metastasectomy when a wedge resection cannot be performed, high risk patients (poor lung function/marginal performance status).

\section{Position and incisions}

The patient is placed under general anaesthesia and in a left or right lateral decubitus position (depending on the site of the operation), with single-lung ventilation via a doublelumen ventilation. The flexion point of the table should be located at the level of the xiphoid process to widen the intercostal spaces. Alternatively, a pillow can be used. We recommend that the upper arm be extended and supported with a padded armrest and soft rolls or a beanbag used to secure the patient on the table. We strongly advise that the same position always be used in order to determine the location of the incision based on similar anatomic references, i.e., inferior margin of the scapula, subxiphoid process, latissimus dorsalis muscle edge and nipple in males or breast line in the women. Moreover, this allows one to have the same orientation to facilitate dissection and insertion of instruments and staplers.

The surgeon could be positioned in front of the patient, with an assistant and a scrub nurse standing at the back and a screen placed at the head of the patient. On the other hand, the assistant can be placed in the same side of the surgeon (right side of the surgeon for upper lobes, left side of the surgeon for lower lobe).

A 3-4 $\mathrm{cm}$ incision is made between the anterior and posterior axillary lines in the fifth or fourth intercostal space, either more anterior or posterior as per the surgeon's preference. Once again, we recommend that the incision always be made in the same place, to develop one's hand- 
eye coordination faster. We advocate the use of a wound protector to facilitate the insertion of the instruments and prevent wound contamination from the cancer. The 30 degrees scope should always be kept in the lateral portion of the wound, creating a space in the medial part to work and perform bimanual instrumentation. It is crucial that to facilitate the operation, the camera assistant hold the camera with one hand and keep the other hand free to actively assist the surgeon in retracting or grasping the lung.

\section{Technical highlights and recommendations}

We recommend that a 5-10 mm HD 30-degree scope and uniportal VATS instruments always be used, as well standard energy devices, such as harmonic devices or LigaSure $^{\mathrm{TM}}$ (Covidien, Medtronic, MN, USA). It is essential that one become used to the uniportal angles during the dissection, to create adequate exposure and identify the anatomic pulmonary structures using a 'swab-on-a-stick' or a thoracoscopic grasper.

The segmental vein, artery and bronchus should be individually dissected, taking care to always include the stumps of these three structures in the segmental resection.

Once the segmental bronchus is identified for dissection, the real anatomy should be compared with the CT scan, in case of any doubt. Hence, we strongly endorse having access to the images inside the operating theatre. Before dividing the segmental bronchus with the Endostapler, we strongly recommend the inflation/deflation method (the segmental bronchus should always be clamped and the anaesthetist asked to insufflate the remaining lung, to ensure that the remaining segments are inflated) (13-15). In some cases, a curved-tip stapler can be used to facilitate the passage around the structures. Furthermore, always including the venous, arterial and bronchial stumps helps with the direction of the staplers. Frequently, during the stapling and division of the intersegmental fissures, one can damage the remaining segmental bronchus, resulting in a bi-segmentectomy or lobectomy. Therefore, we recommend again the inflating deflating method during the stapling of the intersegmental fissure (ensure once again that the remaining segments are still expanding properly and the whole proper segment has been removed).

Regarding dissection and division of vessels including artery and veins, there are various options such as using vascular articulated $30-\mathrm{mm}$ reloads or ligating vessels with a silk tie or clips and subsequently using energy devices. We strongly recommend using Click'a $V^{\circledR}$ Endoscopic Polymer Clip
Appliers 45 degrees (Grena 170 Ltd., Brentford, England); however, other kind of clips can also be useful. For the extraction of the specimen, we suggest using an Endo Catch ${ }^{\mathrm{TM}}$ bag (Covidien, Medtronic, MN, USA) or a similar one.

It is possible to perform all the anatomic segments in both lungs with a uniportal approach. In the coming sections, we will describe the surgical technique and steps to perform the most common segmental anatomic resections.

\section{Surgical technique right side}

\section{Uniportal VATS right upper lobe apical segmentectomy (S1)}

(I) Incision should be placed in the 4th intercostal space. Retract the lung with a 'swab-on-stick' or thoracoscopic grasper to expose the anterior mediastinal pleura. Here, retraction needs to be maintained by the camera assistant or second assistant. With bimanual instrumentation (in left hand, suction and in right hand, dissector or energy device), dissect the hilar structures adequately. The first step is to identify the mediastinal trunk of the artery and the upper vein (Figure 2A,B);

(II) Identify, dissect and divide the apical segmental vein;

(III) Identify, dissect and divide the apical artery (Figure 2C);

(IV) Identify, dissect and divide the apical bronchus with articulated Endostapler and remember, before transecting the bronchus, to always identify the anterior and posterior bronchus and ask the anaesthetist to insufflate the lung to check the correct segmental bronchus is being transected (in some cases a curved-tip stapler technology can be used to facilitate the passage around the structures). During insufflation the portion that remains collapsed is the apical segment (left in Figure 2D);

(V) Pull up the upper lobe with thoracoscopic grasper and complete the intersegmental fissure created after insufflation with Endostapler (right in Figure 2D); always include the stumps of the segmental artery, the vein and the bronchus in the segmental resection;

(VI) Retrieve the resected lung with an Endo Catch ${ }^{\mathrm{TM}}$ bag (Covidien, Medtronic, MN, USA).

\section{Uniportal VATS right upper lobe posterior segmentectomy (S2)}

(I) Incision should be placed in the 4th intercostal 

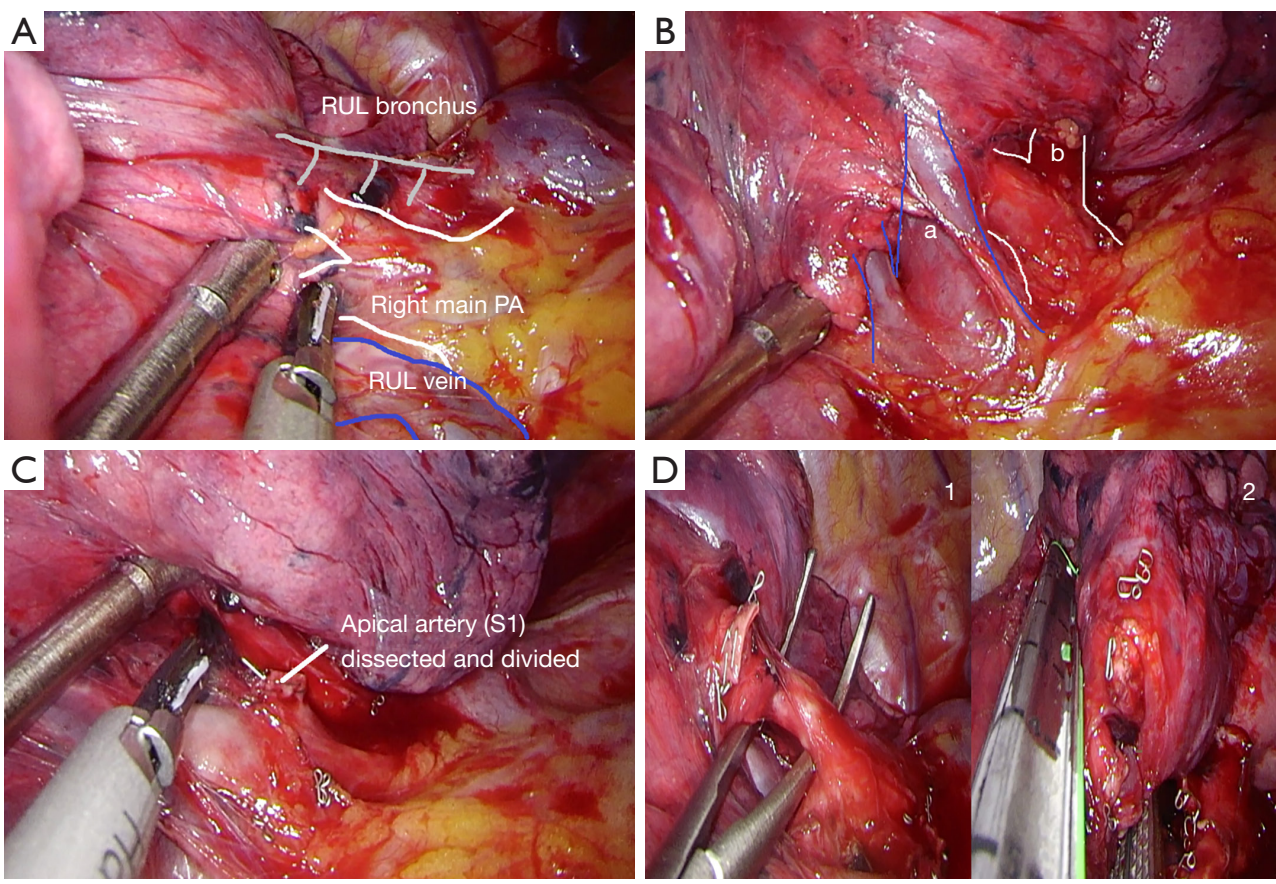

Figure 2 Uniportal VATS right upper lobe apical segmentectomy. (A) Anterior mediastinal pleura exposed with a 'swap-on-stick' or thoracoscopic lung grasper showing the hilar structures including: right main pulmonary artery, right upper vein and right upper bronchus; (B) this image shows the segmental anatomic structures before the dissection: (a) apical segmental vein and (b) apical segmental artery; (C) apical artery divided with stapler; (D) (1) apical bronchus passed with the Endostapler prepared to divide the apical segmental bronchus; (2) completion of the segmental fissure of the apical segment including the stumps of the apical artery, apical vein and apical bronchus. VATS, video-assisted thoracoscopic surgery; RUL, right upper lobectomy; PA, pulmonary artery.

space. Retract the lung with a 'swab-on-stick' or thoracoscopic grasper to expose the fissure (Figure $3 A$ ). The first step is to identify the posterior segmental artery or vein. (In case there is no fissure, the first step is to open the posterior mediastinal pleura and find the posterior segmental bronchus);

(II) Identify, dissect and divide the posterior segmental vein (Figure 3B);

(III) Identify, dissect and divide the posterior segmental artery (Figure 3B);

(IV) Identify, dissect and divide the posterior bronchus with articulated Endostapler. During insufflation the portion that remains collapsed is the posterior segment (Figure 3C);

(V) Complete the intersegmental fissure created after insufflation with Endostapler (Figure 3D);

(VI) Retrieve the resected lung with an Endo CatchTM bag (Covidien, Medtronic, MN, USA).

\section{Uniportal VATS right upper lobe anterior segmentectomy (S3)}

(I) Incision should be placed in the 4th intercostal space, retract the lung with a 'swab-on-stick' or a thoracoscopic grasper to expose the anterior mediastinal pleura and with bimanual instrumentation, dissect the hilar structures adequately. The first step is to identify the mediastinal trunk of the artery and the upper vein;

(II) Identify, dissect and divide the anterior segmental artery (Figure $4 A$ );

(III) Identify, dissect and divide the anterior segmental vein (Figure 4B);

(IV) Identify, dissect and divide the anterior bronchus with articulated Endostapler (Figure 4C);

(V) Complete the intersegmental fissure created after insufflation (Figure 4D);

(VI) Retrieve the resected lung with an Endo Catch ${ }^{\mathrm{TM}}$ bag (Covidien, Medtronic, MN, USA) (Figure 4). 

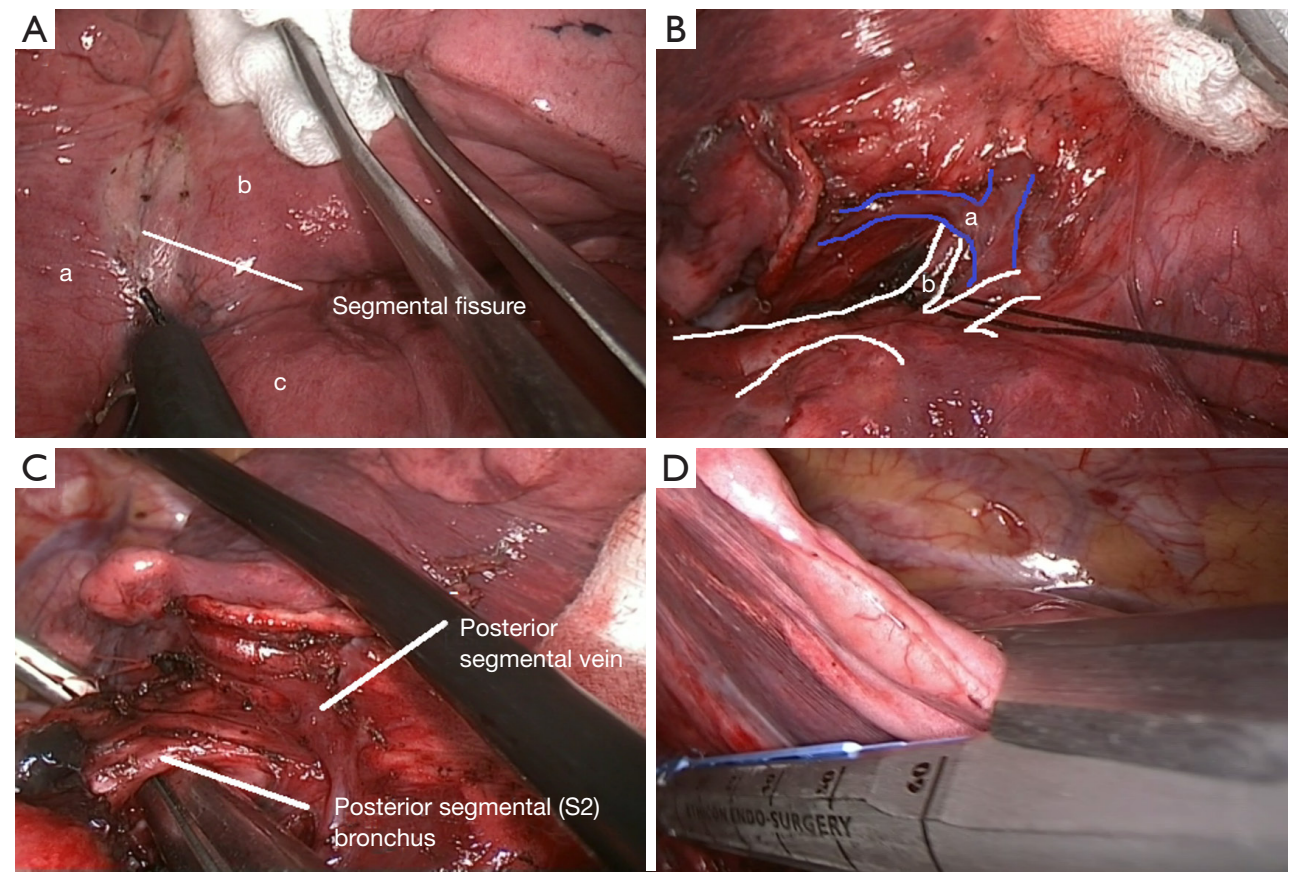

Figure 3 Uniportal VATS right upper lobe posterior segmentectomy. (A) Fissure exposed with bimanual instrumentation (suction and electrocautery) holding up by surgeon and 'swab-on-stick' holding up by the assistant: (a) lower lobe (b) upper lobe (c) middle lobe; (B) fissure exposed and completed with Endostapler: (a) posterior segmental vein in the fissure; (b) posterior segmental artery dissected and looped with a silk tie; (C) posterior segmental bronchus dissected; (D) completion of segmental fissure of the posterior segment including the stumps of the apical artery, apical vein and apical bronchus. VATS, video-assisted thoracoscopic surgery.

\section{Uniportal VATS right lower lobe dorsal segmentectomy (S6)}

(I) Incision should be placed in the 5 th intercostal space. Retract the lung with a 'swab-on-stick' or thoracoscopic grasper to expose the fissure (Figure $5 A$ ). The first step is to identify the dorsal segmental artery or vein (Figure $5 B$ ). In case there is no fissure, the first step is to open the posterior mediastinal pleura and find the dorsal segmental bronchus;

(II) Identify, dissect and divide the dorsal segmental vein;

(III) Identify, dissect and divide the dorsal segmental artery (Figure 5C);

(IV) Identify, dissect and divide the dorsal bronchus with articulated Endostapler (Figure 5D);

(V) Complete the intersegmental fissure created after insufflation with Endostapler;

(VI) Retrieve the resected lung with an Endo Catch TM bag (Covidien, Medtronic, MN, USA).

\section{Uniportal VATS right lower basal segmentectomy (S7-S10)}

(I) Incision should be placed in the 5 th intercostal space, retract the lung with a 'swab-on-stick' or thoracoscopic grasper to expose the fissure and with bimanual instrumentation, identify and dissect the basal segmental artery. Dissect all the basal segmental branches of the artery (S7-S10) and identify the one that should be divided. Always mark the lesion preoperatively with a hook wire or any other technique. With hybrid technology, it is possible to locate the lesion in the same procedure. This may help to facilitate identification of the segment;

(II) Dissect and divide the appropriate basal segmental artery;

(III) Dissect the pulmonary ligament and identify the right lower vein, dissect the distal segmental branches and divide the appropriate basal segmental vein; 

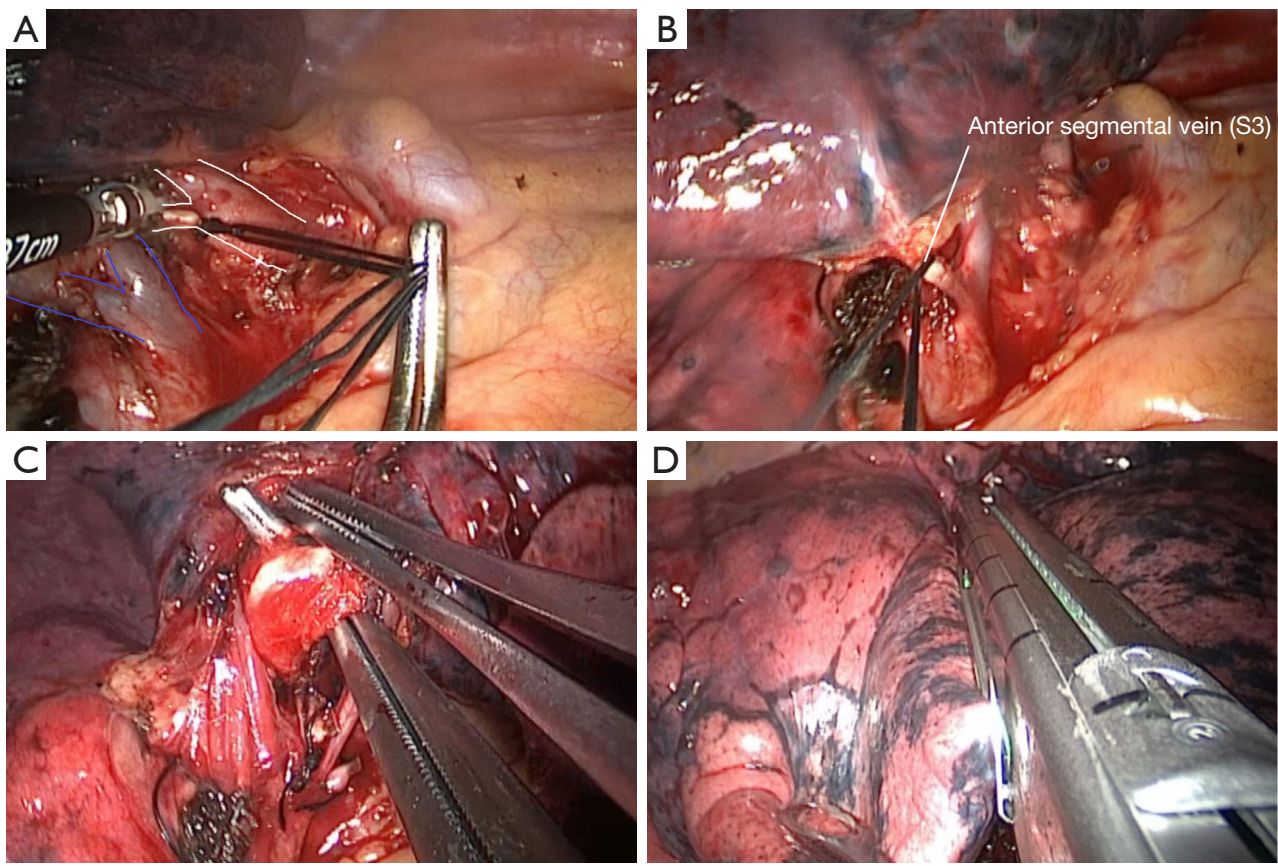

Figure 4 Uniportal VATS right upper lobe anterior segmentectomy. (A) Anterior mediastinal pleura exposed. Identification of anterior segmental artery looped with a silk tie; (B) the image show the anterior segmental vein looped with a silk tie; (C) anterior segmental bronchus dissected with thoracoscopic right angle; (D) completion of segmental fissure of the anterior segment including the stumps of the anterior artery, vein and bronchus. VATS, video-assisted thoracoscopic surgery.

(IV) Behind the divided basal segmental vein, one may now identify, dissect and divide the appropriate basal segmental bronchus with articulated Endostapler. Before transecting the bronchus, always ask the anaesthetist to insufflate the lung to ensure that the correct segmental bronchus is being divided (in some cases a curved-tip stapler technology can be used to facilitate the passage around the structures). During insufflation the portion that remains collapsed is the basal segment;

(V) Pull up the upper lobe with thoracoscopic grasper and complete the intersegmental fissure created after insufflation with the Endostapler;

(VI) Retrieve the resected lung with an Endo CatchTM bag (Covidien, Medtronic, MN, USA) (Figure 6).

\section{Surgical technique left side}

\section{Uniportal VATS left upper lobe trisegmentectomy $(S 1+S 2+S 3)$}

(I) Retract the lung with a 'swab-on-stick' or thoracoscopic grasper to expose the anterior mediastinal pleura and with bimanual instrumentation dissect the hilar structures adequately. The first step is to identify the mediastinal trunk of the left artery and the left upper vein (Figure $7 A$ );

(II) Identify, dissect and divide the tri-segmental vein;

(III) Identify, dissect and divide the tri-segmental artery (Figure 7B);

(IV) By following the vein, it is possible to identify the tri-segmental bronchus. Dissect and divide the trisegmental bronchus with an articulated Endostapler (Figure $7 C$ ); before transecting the bronchus, always identify the lingular bronchus and ask the anaesthetist to insufflate the lung to ensure that the correct segmental bronchus is being divided (in some cases a curved-tip stapler technology can be used to facilitate the passage around the structures). During insufflation the portion that remains collapsed is the tri-segmental segment;

(V) Complete the intersegmental fissure created after insufflation with an Endostapler (Figure 7D);

(VI) Retrieve the resected lung with an Endo CatchTM bag (Covidien, Medtronic, MN, USA). 

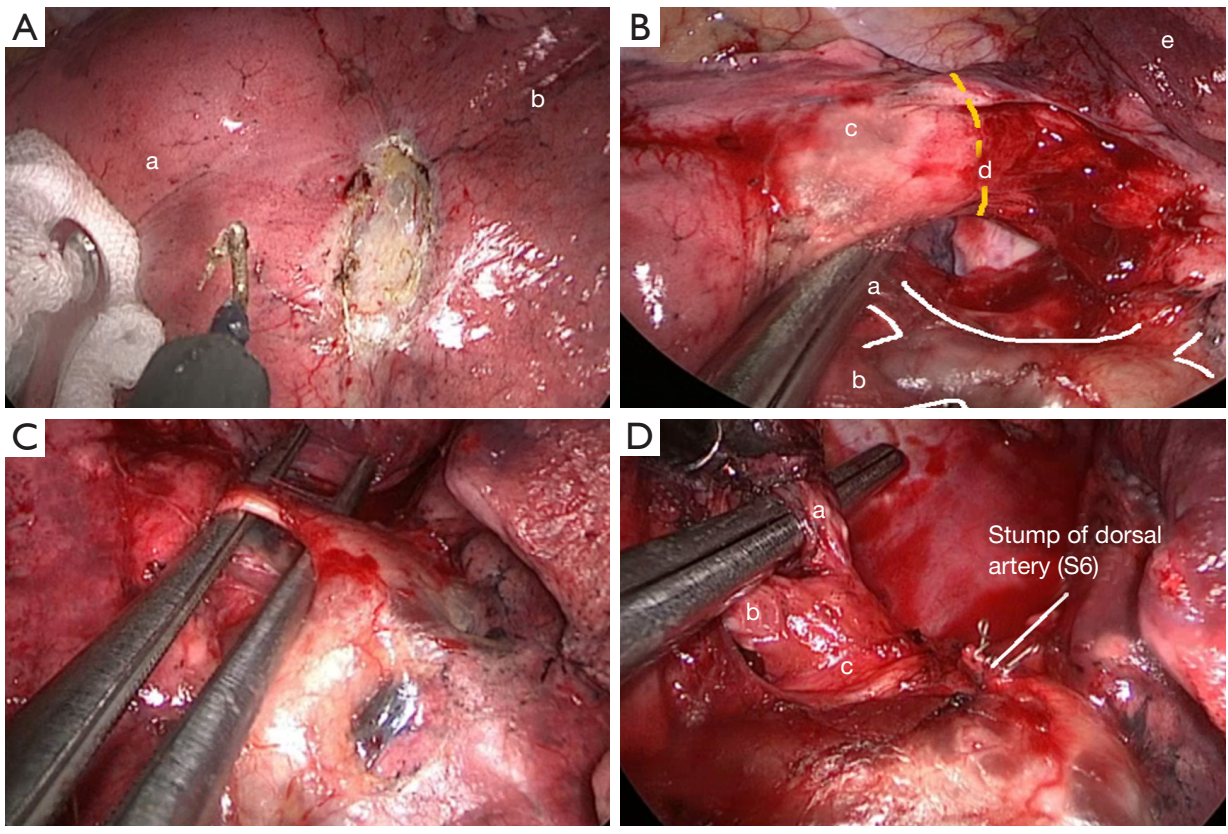

Figure 5 Uniportal VATS right lower lobe dorsal segmentectomy. (A) Fissure exposed with bimanual instrumentation (suction and 'swab-on-stick' holding up by the surgeon: (a) lower lobe dorsal segment, (b) upper lobe posterior segment; (B) fissure dissected between upper and lower lobe: (a) right dorsal segmental artery, (b) basal segmental artery, (c) dorsal segment, (d) fissure between dorsal segment right lower lobe and posterior segment right upper lobe, (e) posterior segment right upper lobe; (C) dorsal segmental artery dissected; (D) right lower lobe bronchus dissected: (a) dorsal segmental bronchus, (b) basal segmental bronchus, (c) right lower lobe main bronchus. VATS, video-assisted thoracoscopic surgery.
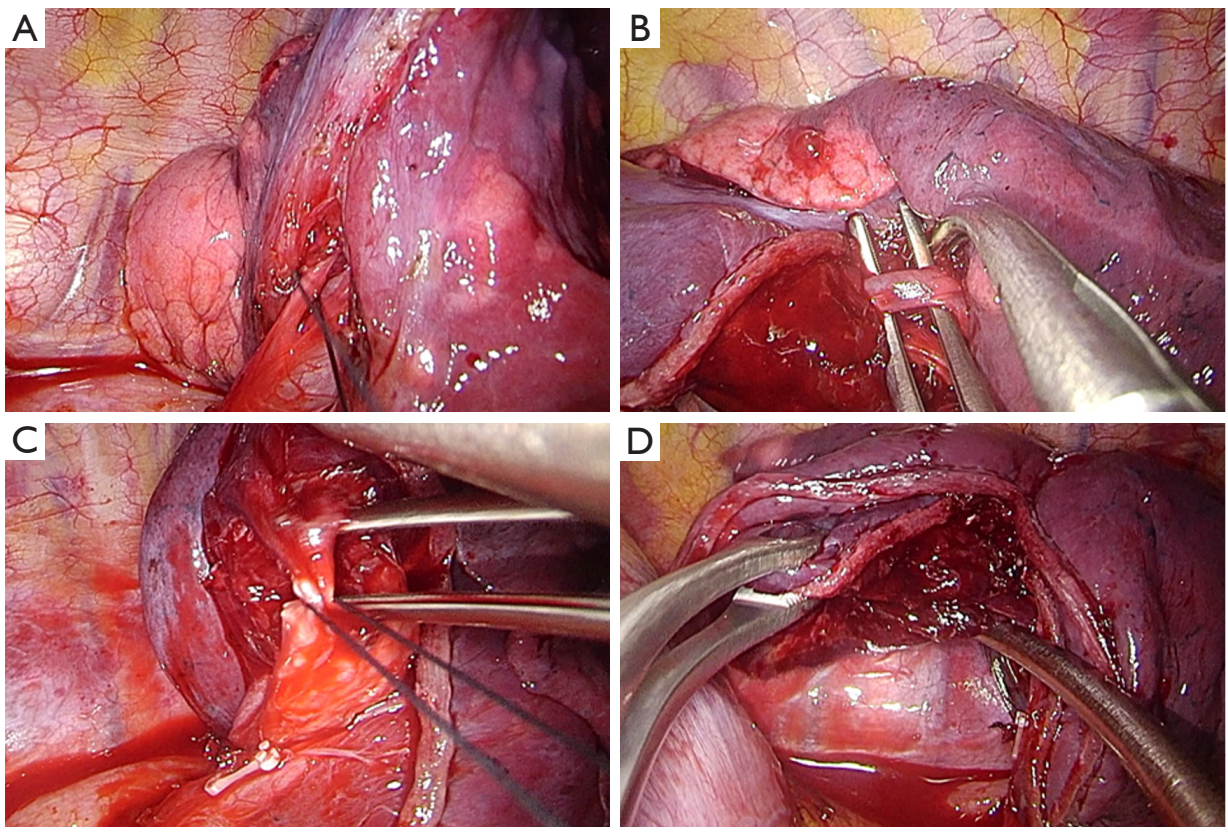

Figure 6 Uniportal VATS right lower basal segmentectomy. (A) Dissection of the right lower pulmonary ligament, location of lower vein and dissection of the postero-basal vein; (B) fissure opened and exposed the basal artery, postero-basal artery dissected; (C) after cut the postero-basal artery the bronchus is behind, this image shows the postero-basal segment bronchial segment; (D) completion of the fissures with Endostapler. VATS, video-assisted thoracoscopic surgery. 

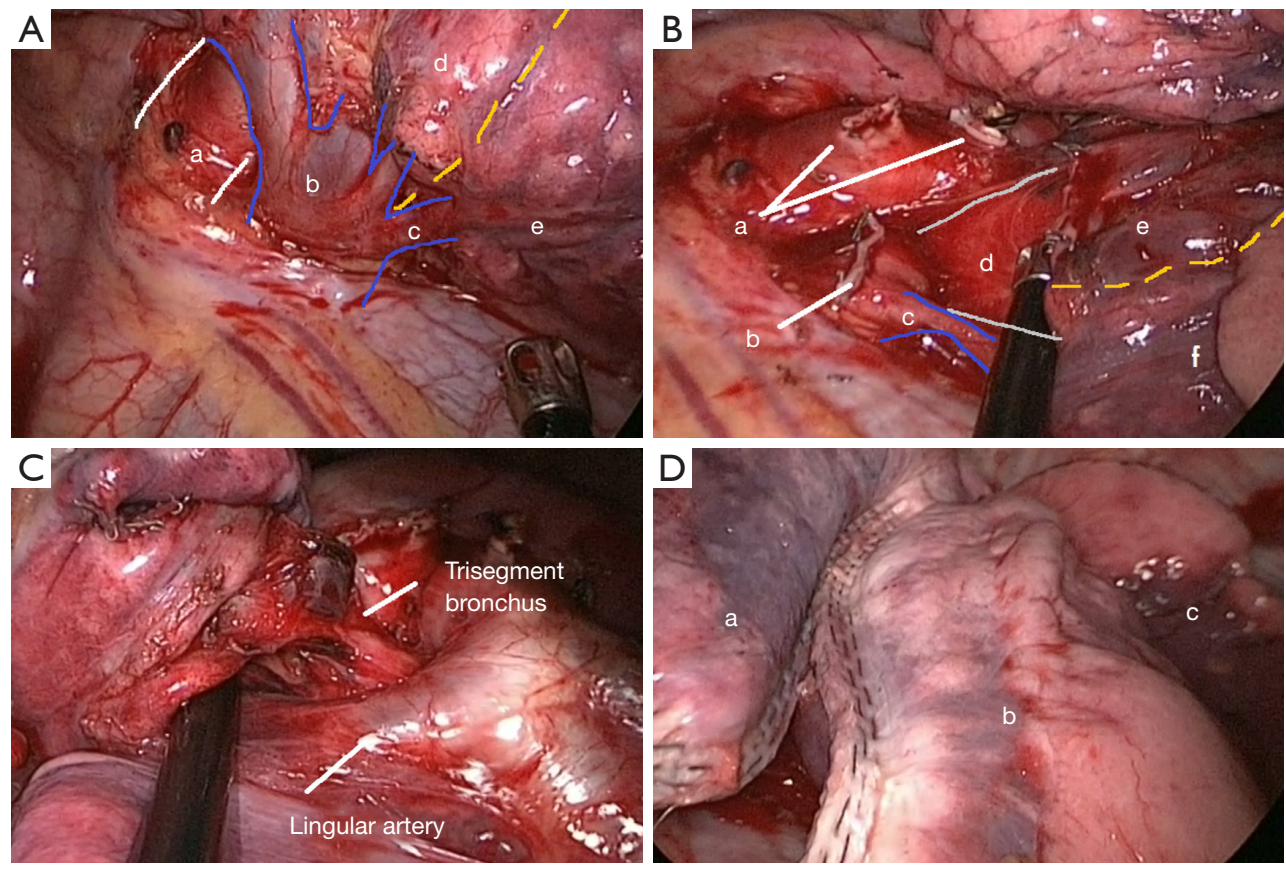

Figure 7 Uniportal VATS left upper lobe trisegmentectomy. (A) Anterior mediastinal pleura exposed with a 'swap-on-stick' or thoracoscopic lung grasper showing the hilar structures including: (a) left main pulmonary artery, (b) tri-segmental vein, (c) lingular vein, (d) tri-segment, (e) lingular segment; (B) (a) tri-segmental artery segmental branches resected (b) tri-segmental vein divided with vascular Endostapler (c) lingular vein (d) left main bronchus (e) tri-segment (f) lingula segment; (C) tri-segmental bronchus dissected and ready to be cut with Endostapler; (D) completion of the intersegmental fissure (a) tri-segment (b) lingula segment (c) left lower lobe. VATS, video-assisted thoracoscopic surgery.

\section{Uniportal VATS left upper lobe lingulectomy (S4-S5)}

(I) Retract the lung with a 'swab-on-stick' or thoracoscopic grasper to expose the anterior mediastinal pleura and with bimanual instrumentation, dissect the hilar structures adequately. The first step is to identify the mediastinal trunk of the left artery and the left upper vein;

(II) Identify, dissect and divide the lingular vein (Figure 8A);

(III) Identify, dissect and divide the lingular artery (Figure 8B);

(IV) By following the vein, it is possible to identify the lingular bronchus. Dissect and divide the lingular bronchus with an articulated Endostapler (Figure 8C). Complete the intersegmental fissure created after insufflation, with the Endostapler (Figure 8D);

(V) Retrieve the resected lung with an EndoCatch ${ }^{\mathrm{TM}}$ bag (Covidien, Medtronic, MN, USA).

\section{Comments}

Once you gain experience in the approach, it is essential to repeat the same steps consistently multiple times in order to hone the surgical skill. Uniportal VATS approach for anatomical segmentectomies is feasible and safe. If one is planning to perform segmental anatomical resections, we highly recommend that the surgeon has passed the learning curved and experienced a large volume of cases, familiarised themselves with the segmental anatomy and initially perform the surgery in carefully selected cases.

In cases where the lesion is too deep to be palpated, it is possible to mark the lesion preoperatively. We strongly recommend marking the lesion with a hook wire (Figure 9). But there are other very effective methods like insertion of coils, injected radiopaque material by CT guidance, Electromagnetic navigational bronchoscopy (16), C-arm fluoroscopy (17) or hybrid technology that has been proven to be helpful to locate the 

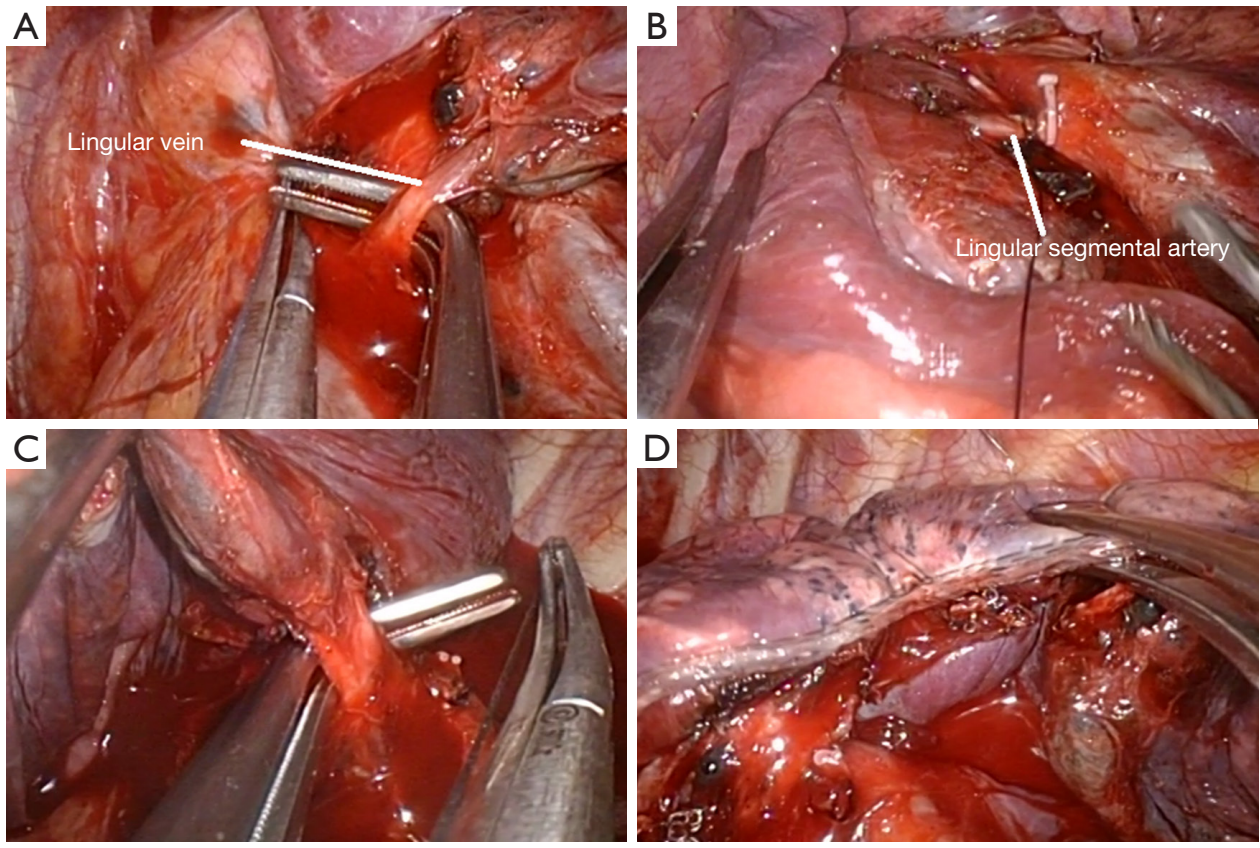

Figure 8 Uniportal VATS left upper lobe lingulectomy. (A) Anterior mediastinal pleura exposed with a 'swap-on-stick' or thoracoscopic lung grasper showing the hilar structures including the lingular segmental vein dissected; (B) lingular segmental artery ligated with a silk tie and a clip and prepared to de resected with energy device; (C) lingular segmental bronchus dissected in the fissure; (D) completion of the intersegmental fissure between tri-segment and lingular segment including the stumps of the artery, vein and bronchus. VATS, video-assisted thoracoscopic surgery.
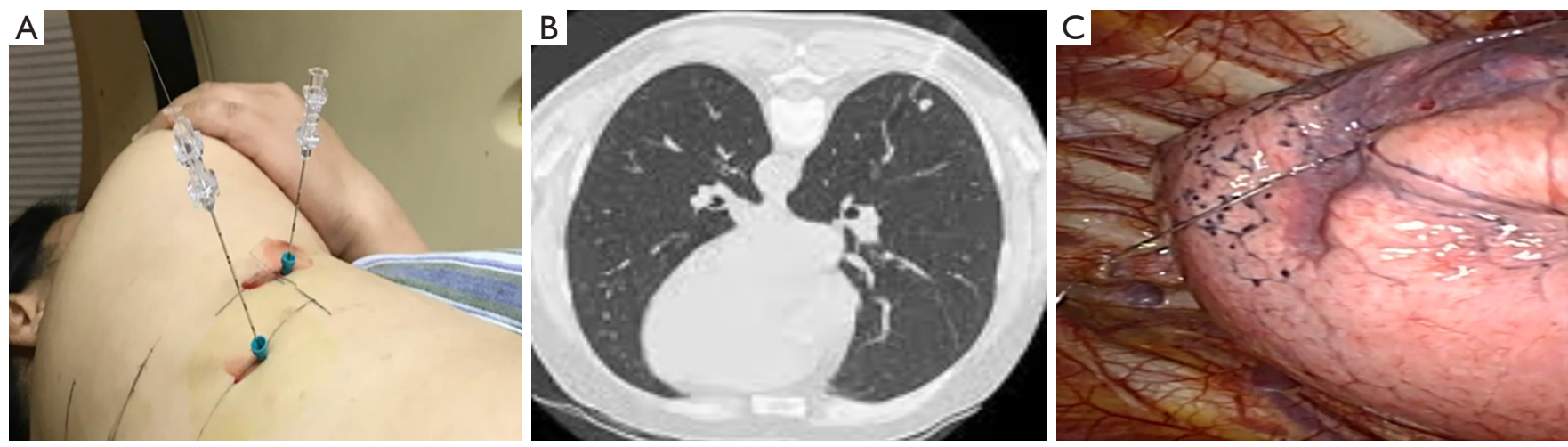

Figure 9 Image shows the location of the lesion with how wire, views in the patient, in the CT scan and in the VATS view. VATS, videoassisted thoracoscopic surgery; CT, computed tomography.

lesion in the same procedure.

In some cases where the lesion is in the intersegmental fissure, a bi-segmentectomy can be performed. This happens more commonly in the upper lobes. Furthermore after passed the basic learning curve of uniportal VATS segmentectomies, every segment of the right, upper and middle lobe are able to be performed, including two segments of the same lobe or different lobes.

Uniportal VATS segmentectomy is a demanding technique, safe and feasible in selected patients with good results in the literature (18-20) that seems to be an acceptable alternative to conventional VATS or open thoracotomy when the surgeon completed the training period and learning curve. 


\section{Acknowledgements}

None.

\section{Footnote}

Conflicts of Interest: The authors have no conflicts of interest to declare.

\section{References}

1. Anile M, Diso D, De Giacomo T. Uniportal thoracoscopic lobectomy. Ann Thorac Surg 2013;96:745.

2. Ng CS. Uniportal VATS in Asia. J Thorac Dis 2013;5 Suppl 3:S221-5.

3. Hernandez-Arenas LA, Lin L, Yang Y, et al. Initial experience in uniportal subxiphoid video-assisted thoracoscopic surgery for major lung resections. Eur J Cardiothorac Surg 2016;50:1060-6.

4. Harris CG, James RS, Tian DH, et al. Systematic review and meta-analysis of uniportal versus multiportal videoassisted thoracoscopic lobectomy for lung cancer. Ann Cardiothorac Surg 2016;5:76-84.

5. Gonzalez-Rivas D, Fernandez R, Fieira E, et al. Uniportal video-assisted thoracoscopic bronchial sleeve lobectomy: first report. J Thorac Cardiovasc Surg 2013;145:1676-7.

6. Gonzalez-Rivas D, Delgado M, Fieira E, et al. Single-port video-assisted thoracoscopic lobectomy with pulmonary artery reconstruction. Interact Cardiovasc Thorac Surg 2013;17:889-91.

7. Nakayama H, Yamada K, Saito H, et al. Sublobar resection for patients with peripheral small adenocarcinomas of the lung: surgical outcome is associated with features on computed tomographic imaging. Ann Thorac Surg 2007;84:1675-9.

8. Asamura H. Minimally invasive approach to early, peripheral adenocarcinoma with ground-glass opacity appearance. Ann Thorac Surg 2008;85:S701-4.

9. Schuchert MJ, Pettiford BL, Keeley S, et al. Anatomic segmentectomy in the treatment of stage I non-small cell lung cancer. Ann Thorac Surg 2007;84:926-32; discussion 932-3.

10. Martin-Ucar AE, Nakas A, Pilling JE, et al. A case matched study of anatomical segmentectomy versus lobectomy for stage I lung cancer in high risk patients. Eur J Cardiothorac Surg 2005;27:675-9.

11. Koike T, Yamato Y, Yoshiya K, et al. Intentional limited pulmonary resection for peripheral T1 N0 M0 small-sized lung cancer. J Thorac Cardiovasc Surg 2003;125:924-8.

12. Smith CB, Swanson SJ, Mhango G, et al. Survival after segmentectomy and wedge resection in stage I non-smallcell lung cancer. J Thorac Oncol 2013;8:73-8.

13. Okada M, Yoshikawa K, Hatta T, et al. Is segmentectomy with lymph node assessment an alternative to lobectomy for non-small cell lung cancer of $2 \mathrm{~cm}$ or smaller? Ann Thorac Surg 2001;71:956-60; discussion 961.

14. Okada M, Mimura T, Ikegaki J, et al. A novel video assisted anatomic segmentectomy technique: selective segmental inflation via bronchofiberoptic jet followed by cautery cutting. J Thorac Cardiovasc Surg 2007;133:753-8.

15. Tsubota N. An improved method for distinguishing the intersegmental plane of the lung. Surg Today 2000;30:963-4.

16. Pittet O, Christodoulou M, Pezzetta E, et al. Videoassisted thoracoscopic resection of a small pulmonary nodule after computed tomography-guided localization with a hook-wire system. Experience in 45 consecutive patients. World J Surg 2007;31:575-8.

17. Kim HK, Choi YH. The feasibility of single-incision video-assisted thoracoscopic major pulmonary resection performed by surgeons experienced with a twoincision technique. Interact Cardiovasc Thorac Surg 2015;20:310-5.

18. Gonzalez-Rivas D, Fieira E, Mendez L, et al. Single-port video-assisted thoracoscopic anatomic segmentectomy and right upper lobectomy. Eur J Cardiothorac Surg 2012;42:e169-71.

19. Wang BY, Liu CY, Hsu PK, et al. Single-incision versus multiple-incision thoracoscopic lobectomy and segmentectomy: a propensity-matched analysis. Ann Surg 2015;261:793-9.

20. Han KN, Kim HK, Choi YH. Uniportal videoassisted thoracoscopic surgical (VATS) segmentectomy with preoperative dual localization: right upper lobe wedge resection and left upper lobe upper division segmentectomy. Ann Cardiothorac Surg 2016;5:147-50.

Cite this article as: Hernandez-Arenas LA, Purmessur RD, Gonzalez-Rivas D. Uniportal video-assisted thoracoscopic segmentectomy. J Thorac Dis 2018;10(Suppl 10):S1205-S1214. doi: 10.21037/jtd.2018.02.47 